\title{
ASIAN AMERICANS AND MENTAL HEALTH: Cultural Barriers to Effective Treatment
}

HEATHER SPELLER

DISPARITIES IN MENTAL HEALTH CARE FOR RACIAL MINORITIES REMAINS A SERIOUS AND VERY REAL PROBLEM CALLING FOR IMMEDIATE ATTENTION. THE 2001 REPORT OF THE SURGEON GENERAL AFFIRMED THAT ETHNIC AND RACIAL MINORITIES HAVE LESS ACCESS TO AND AVAILABILITY OF MENTAL HEALTH SERVICES, AND ARE SUBSEQUENTLY LESS LIKELY TO RECEIVE NEEDED MENTAL HEALTH SERVICES. THIS PAPER EXAMINES A RANGE OF ISSUES REGARDING ASIAN AMERICAN MENTAL HEALTH. IT PRESENTS THE PRACTICAL AND CULTURAL BARRIERS THAT MEMBERS OF THIS ETHNIC GROUP CONFRONT WHEN SEEKING MENTAL HEALTH CARE AND EXPLAINS HOW CULTURAL DIFFERENCES SOMETIMES RESULTS IN MISDIAGNOSIS AND INEFFECTIVE TREATMENT. IT ALSO EXPLORES WAYS THAT THE AMERICAN MENTAL HEALTH CARE SYSTEM CAN IMPROVE TO ACCOMMODATE DIVERSE ETHNIC GROUPS. 


\section{"Asian Americans frequently experience and express mental illness very differently from Westerners, often emphasizing somatic rather than psycho- logical symptoms. These variations can often render accurate assessment and diagnosis more difficult for mental health professionals..."}

Asian Americans are the fastest growing minority group in the United States today. According to the 200I U.S. Census, there are over 10.2 million Asian Americans in the U.S., making up 3.6 percent of the total population. It is estimated that by 2020 Asian Americans will number approximately 20 million, or 6 percent of the population. Within the current Asian American population 62 percent are foreign born, however American born Asian Americans are expected to outnumber immigrants by the year 2020. ${ }^{i}$ As the most diverse ethnic group in the U.S., the heterogeneity among Asian Americans is staggering. There are as many as 43 different Asian American ethnic groups, each with distinct cultural values, religious traditions, dietary practices, histories, and over Ioo languages. ${ }^{\text {ii }}$

Over the past few decades there have been well-documented mental health disparities among Asian Americans. Members of this ethnic group have been found to experience a number of difficult practical and cultural barriers to effective and culturally appropriate mental health care. Asian Americans frequently experience and express mental illness very differently from Westerners, often emphasizing somatic rather than psychological symptoms. These variations can often render accurate assessment and diagnosis more difficult for mental health professionals who are unfamiliar with Asian culture and patterns in Asian American mental health. However these difficulties and disparities can be overcome by increasing cultural compe- tency and making ethnic specific services available to Asian American communities.

\section{ASIAN AMERICAN}

\section{MENTAL HEALTH DISPARITIES}

The model minority myth has promoted the stereotype that Asian Americans are successful, hard-working, and intelligent. They are frequently viewed as resilient and healthy, experiencing fewer mental health problems than other groups. It is often assumed that the disproportionately small number of Asian Americans utilizing mental health services is simply an effect of an overall lower incidence of mental illness. However, low demand for mental health care is not necessarily reflective of low need.iii These, and other erroneous conclusions stemming from the model minority myth, are just that: myths.

In reality, Asian Americans do experience mental illness and have an equally high, if not higher, need for appropriate mental health services as any other racial or ethnic group. Asian American adolescent boys are twice as likely to have been physically abused and are three times more likely to report abuse than their white American counterparts.iv Asian American women aged 15-24 and 65+ have the highest suicide rates in the U.S. out of all racial and ethnic groups. ${ }^{\vee}$ Asian American college students have been found to report higher levels of depressive symptoms than white studentsvi and Kessler and others found that Chinese 
Americans have a lifetime prevalence of major depression of as high as I7 percent (I994). Immigrants and refugees are considered to be "high-need" populations, as a result of magnified stressors and difficulties frequently experienced in a new and unfamiliar culture. Some of the common reported stressors include crime and personal safety, unemployment/financial difficulties, language barriers, physical illness, and lack of a support network of friends and relatives, vii

A consistent pattern of underutilization of mental health services among Asian Americans has been well documented for several decades. viii Those who do receive mental health treatment are often greatly delayed in help-seeking, and thus tend to be more severely ill upon treatment initiation. ${ }^{\text {ix }}$ Such phenomena likely contribute to the findings that Asian Americans who do receive inpatient care necessitate more intensive treatment and have comparatively longer lengths of stay. ${ }^{\mathrm{x}}$ Poor outcomes for short-term treatment are also common, with decreased patient satisfaction and frequent premature termination of care. ${ }^{\mathrm{xi}}$ In I998 Zhang and others found that Asian Americans, when compared to White Americans, were much less likely to report mental health problems to friends or relatives, psychiatrists or mental health specialists, or to physicians (with relative rates of $\mathrm{I} 2$ percent, 4 percent, and 3 percent).

\section{BARRIERS TO SEEKING HELP}

PRACTICAL BARRIERS

Several barriers to mental health treatment for Asian Americans have been identified, all of which are thought to contribute to these problematic trends of underutilization. Limited availability is one of the important issues that must be addressed. In 1998 Manderscheid and Henderson found there were approximately 70 Asian American providers available per 100,000 Asian Americans in the U.S., with a ratio of over twice that for whites. The availability of bilingual Asian American providers is crucial, especially among immigrant populations for whom language presents a huge barrier to care. Other practical barriers include high costs of treatment, a problem only magni- fied by the fact that over 2 I percent of Asian Americans lack health insurance and as many as I 4 percent are living below the poverty level.xii Many individuals working one or two full-time jobs experience difficulties with scheduling and time constraints, unable to take time off of work. However, one of the greatest barriers is a general lack of mental health awareness, especially regarding what services are available and how to access them. xiii

\section{CULTURAL BARRIERS}

Perhaps even more significant than the practical barriers are the cultural barriers that frequently deter Asian Americans from seeking psychological help. However, in order to appreciate and effectively address these cultural barriers, it is first necessary to recognize the vast cultural differences between traditional Asian values and those of the dominant American culture. Asian values are centered on the concept of interdependence in a collectivistic society. As a result Asian cultures emphasize concepts such as emotional self-control, humility, filial piety, family recognition through achievement, and the integration of the mind and body. Contrastingly, American society glorifies the individual, encouraging the self-sufficiency and independence. American culture tends to value emotional self-expression, expression of pride, a duty to satisfy personal needs, self-recognition through achievement, and the separation of mind and body. xiv

Subsequently it should not be surprising that many Asian Americans have considerable conceptual difficulties regarding the western notions of mental illness and mental health services. Individuals who embrace the theory of mind-body holism often experience great difficulty distinguishing between psychological and physical ailments. ${ }^{\mathrm{xv}}$ However, in order to successfully navigate the American mental health system, it is imperative to have a clear conceptualization of "mental illness" and how it and its associated symptoms differ from physiological illness.

Furthermore, Asian Americans who do have a understanding of the Western concept of mental illness often 
view it very negatively, and thus hesitate to utilize mental health services, even if available. In accordance with the cultural tendency towards emotional self-control, many Asian Americans view emotional distress as a sign of weakness, resulting from a lack of discipline or will power. A I976 study of Chinese, Japanese, and Filipino students found that the majority believed good mental health could be achieved by the avoidance of morbid thoughts, and that getting help with this "weakness" reflects poorly on char-acter.xvi Then, in 1983, Cheung and others confirmed that the causes that one attributes to mental illness greatly influence the sources of psychological help one deems appropriate.

One of the greatest cultural barriers to treatment, however, results from the low social acceptability of mental disorders in Asian cultures. Asian Americans frequently view mental illness as highly stigmatizing and thus are less likely to recognize, acknowledge, or seek help for mental health problems. ${ }^{x v i i}$ It is not uncommon for an individual who is experiencing a decreased level of functioning to express feelings of shame and personal failure. He or she might be apprehensive to seek help outside of the family for fear of disgracing the family name, but also be reluctant to discuss mental health issues with family members, for fear of being burdensome.

\section{EXPRESSION OF MENTAL ILLNESS}

SOMATIZATION

Studies have found that this cultural stigma associated with mental illness is a factor influencing the observed tendency towards somatization among Asian Americans. ${ }^{\text {xviii }}$ It is possible that members of this ethnic group are consciously or unconsciously denying the experience and expression of emotions and psychological symptoms, instead expressing such symptoms in more individually and culturally acceptable ways. Many initial theories attributed somatization tendencies to a general lack of psychological mindedness among Asian Americans, exacerbated by the lack of descriptive psychological vocabulary in Asian languages. ${ }^{x i x}$ However studies have since refuted the idea that Asian
Americans have some sort of inability to experience, access, or process psychological difficulties. In I9 82 Cheung and Lau found that Asian American patients are often fully aware of their own emotional problems as well as stressors and chronic difficulties that could be related to both their psychological and somatic symptoms. For example in initial clinical interviews Vietnam refugees reported mostly somatic complaints, however, when they were asked specifically, they had no difficulties identifying and reporting their psychological symptoms as well. ${ }^{x x}$ It was concluded that Asian Americans were likely to selectively present their symptoms based on what they believed to be appropriate for a given clinical setting.

Different styles of symptom expression and a tendency towards somatization can make it much more difficult to recognize and treat mental illness in Asian Americans using traditional Western diagnostic strategies. Western mental health systems are grounded in the mind-body dichotomy, a concept that influences nearly every aspect of health care in America, including the expression of distress. It would not be unusual for an individual accustomed to the traditional Asian organ-oriented concept of pathology to attribute mental distress to bodily disharmony, thus increasing the likelihood of seeking help from a primary care physician rather than a mental health professional. This makes it even more critical to ensure that all Asian Americans have access to culturally sensitive healthcare providers, who are aware of such complexities.

\section{CULTURE-BOUND SYNDROMES}

There are currently many difficulties regarding the crosscultural assessment and diagnosis of mental disorders, as is illustrated by the ongoing debates regarding "culturebound syndromes." A culture-bound syndrome is a recurrent, locality-specific pattern of aberrant behavior and troubling experience that may or may not be linked to a particular diagnostic category.xxi These syndromes are often indigenously considered to be "illnesses" or afflictions, most with local names. Diagnostic and treatment difficulties arise when a disorder that is well-established and 
recognized within one culture remains ambiguous and unclassified in another.

"Neurasthenia" (a.k.a. Shenjing shaijo) is a culture-bound syndrome originating in China that resembles major depressive disorder. However it is often characterized more by salient somatic features and often lacks the depressed mood that is so central to a diagnosis of major depressive disorder, ${ }^{x x i i}$ as classified by the Diagnostic and Statistical Manual of Mental Disorders, Version 4, Text Revision (DSM-IV-TR). Although neurasthenia is classified in the International Classification of Diseases, Version Io, it is not recognized as an official diagnostic category in the DSM-IV-TR. A I997 study found approximately 7 percent of Chinese Americans in Los Angeles to be experiencing symptoms of neurasthenia, less than half of whom were symptomatic of a co-morbid DSM-III disorder.xxiii Findings such as these suggest that culture-bound syndromes are indeed very real phenomena that require further research and clinical attention.

\section{CLINICAL CHALLENGES \& CULTURAL COMPETENCY}

Given the tendency for different ethnic groups to express distress in unique and varied ways, we must ensure that the diagnostic tools being used for assessment adequately account for such potential cultural differences. Unfortunately, misdiagnosis is much more common in crosscultural situations, and it is unclear whether the current Western diagnostic systems and assessment techniques fit with Asian American sub-cultures. ${ }^{\text {xxiv }}$ Assessment tools frequently become even more invalidated when translated into other languages in an attempt to accommodate nonEnglish speaking populations. ${ }^{\mathrm{xxv}}$

Inaccurate mental health evaluation of Asian Americans can also result from cultural biases among clinicians. Mental health professionals who practice rigid adherence to DSM-IV diagnostic criteria may have a narrow way of defining what disorders exist and how they are manifested. It is important to note however, that errors can be a result of overpathologizing or underpathologizing.xxvi Overpathologizing can occur when a clinician who is unfamiliar with the cultural nuances of the patient judges normal cultural variations in beliefs or behaviors as indicative of psychopathology. For example, the experience of hallucinations during religious practices is considered normative in certain cultures. However if the clinician is unaware of these cultural norms, he or she might misdiagnosis the patient with a psychotic disorder. On the other side of the spectrum, the indiscriminate application of cultural explanations to all mental and/or behavioral deviations is termed underpathologizing. This could occur if an Asian American patient's reserved and flat affect is incorrectly attributed to cultural behavior norms instead of to the existence of a depressive disorder. Similar problems can arise if a clinician is influenced by the stereotype of the model minority myth; Asian American clients could be seen as experiencing few or no social and psychological problems, especially in terms of their ability for adjustment to the U.S.

There are many other challenges and traps that may arise when providing mental health care to Asian Americans that a culturally sensitive therapist must be sure to avoid. References to other Asian American sub-groups could offend a client who does not see him or herself as connected (or may actually harbor feelings of hostility) to the other ethnic group.xxvii Given the enormous diversity between the many Asian American ethnic groups, mental health professionals must be aware of the erroneous conclusions that can be made when Asian Americans are treated as a single category. Rather, it is important to recognize diversity within racial and ethnic groups, in addition to being aware of between group differences.

To further complicate these issues there is also a wide range of variation within specific subgroups, due to the varying levels of acculturation among individuals. Acculturation is defined as the adoption of the worldviews and living patterns of a new culture; xxviii it is the process of change that occurs when two or more cultures come into contact. Studies have found that more highly acculturated Asian Americans have more positive attitudes toward seeking 
psychological services, and display higher help-seeking behaviors. xxix They also tend to be more tolerant of the stigma associated with psychological help, and are most open to discussing mental health problems with a psychologist. $\mathrm{xxx}$ There are large variations in values, attitudes, and methods of expressing mental illness between Asian Americans of high and low acculturation, and it must be cautioned that generation (i.e. how long one's family has been in the country) is not always a direct or accurate indicator of acculturation. As a crucial component of providing culturally competent and appropriate care, mental health professionals must be aware of the varying acculturation levels among their own patients.

\section{ETHNIC SPECIFIC SERVICES}

A culturally competent mental health system incorporates skills, attitudes, and policies to ensure that it is effectively addressing the treatment and psychosocial needs of consumers and families with diverse values, beliefs, and sexual orientations, in addition to backgrounds that vary by race, ethnicity, religion, and/or language. One of the most propulsive forces in the struggle for cultural competency is the establishment of ethnic specific services. These services recognize need for more culturally responsive mental health care for ethnic communities, and thus seek to provide bicultural and bilingual personnel, culturally relevant treatment practices, and a culturally familiar and comfortable atmosphere. Studies have found that Asian Americans show increased utilization of mental health services when patient centers are specifically oriented towards Asian Americans, ${ }^{x x x i}$ Lin and Cheung (1999) observed a decrease in delay between symptom onset and help-seeking, as well as a decrease in premature termination of care when ethnic specific services were available.

Another benefit of ethnic specific services is that Asian Americans are more likely to receive culturally appropriate therapeutic techniques, which can have a substantial impact on the treatment outcome and patient satisfaction. For example, one of the most common therapeutic techniques in Western medicine is psychotherapy. This "talking therapy" is often a completely foreign idea to Asian Americans, and is largely at odds with traditional Asian beliefs. Asian American patients are often uncomfortable with the concept of examining and discussing one's inner thoughts and feelings, especially given the commonly held Asian belief that the best way to deal with mental illness is to avoid morbid thoughts and repress emotions. ${ }^{x x x i i}$ The therapeutic technique of cognitive-behavioral therapy has been found much more effective than psychoanalytic techniques among Asian Americans; they often respond better to a directive, pragmatic, problem-solving approach that offers immediate and tangible help.

Cultural sensitivity is even important at the pharmacologic level. Studies have shown Asian Americans to be slow metabolizers of cytochrome P-450 enzymes, rendering them much more sensitive to pharmacotherapy.xxiii Especially for patients who tend to have a smaller body size, it is imperative for clinicians to be aware of these differences and adjust medication doses accordingly.

Despite the many benefits of ethnic specific services as discussed above, some clinicians have questioned possible drawbacks to the establishment of these services. Uba (1982) suggested that such downsides could include an inability to reach Asian Americans outside of major urban areas, and that focusing on cultural competency within specific mental health centers could potentially limit the opportunity to provide culturally competent care among a wider range of service providers. There is also a concern that when Asian Americans become accustomed to using ethnic specific services, they may actually become further discouraged from utilizing other universal services for which cultural specificity is unavailable. It must also be noted that matching Asian Americans with Asian American therapists is not always helpful, especially if the two are from different ethnic subgroups. ${ }^{\text {xxxiv }}$ Depending on factors such as acculturation, personal beliefs, and cultural influences, some Asian Americans actually express a preference for non-Asian American providers. In 2002 Kim and Atkinson found that Asian American clients with high adherence to Asian cultural values evaluated Asian American counselors as more empathetic and credible, 
and clients with low adherence to Asian cultural values evaluated European/American counselors as more empathetic. However somewhat unexpectedly, clients overall rated the session with a European/American counselor as more positive and arousing than the session with an Asian American counselor.

\section{SOUTH COVE COMMUNITY \\ HEALTH CENTER}

The South Cove Community Health Center is an ethnic specific service located in Boston that targets the Asian Americans population in and around Chinatown. I had the opportunity to chat with Dr. Albert Yeung, a clinician in the Behavioral Health Department at South Cove, to inquire about his views on culturally specific clinics. He is a strong advocate, describing the need to reach out to a population who lack a Western conception of mental health, maintain stigmatized attitudes, and thus avoid mental health services. Dr. Yeung was also quick to refute the aforementioned uncertainties and questioned disadvantages, providing a strong case for ethnic specific services. In areas such as Boston's Chinatown where much of the population is composed of non-English speaking immigrants and refugees, many of the people feel as if they cannot and consequently will not seek help, medical or psychological, from the large Boston hospitals. They are anxious, have difficulties navigating the massive hospital mazes, cannot adequately explain their symptoms, and often feel uncomfortable and hesitant to disclose their problems, especially if an interpreter is being used.

South Cove does not force itself on the residents, but is there as a place where members of the Asian community can feel safe and comfortable, and are able to speak with clinicians in their native tongue. Additionally, Dr. Yeung asserted that the use of this culturally specific service does not handicap people, nor does it discourage them from using other non-ethnic specific services in the future. In fact, patients typically "graduate" to mainstream hospitals as their English begins to improve and they become more comfortable with American culture and institutions. Dr. Yeung also refuted the criticism that centers like South Cove limit the opportunity for other services to increase their cultural competency. If anything, centers like South Cove can be used as a model for other mental health centers, demonstrating effective ethnic specific methods of providing quality care to Asian American populations.

However, this is an extremely complex issue. In order to provide the much needed culturally sensitive care, clinicians need to both recognize the illness and form a connection with the patient so that the patient feels comfortable receiving treatment. One commonly held belief is that this should be facilitated by being clear and straightforward, carefully explaining to the patient everything that is going on with the diagnosis, treatment, etc. However Dr. Yeung brought up a difficult ethical issue that must be addressed. It is a frequent practice for doctors in Asian countries to withhold certain pieces of information that they believe could have a detrimental effect on the patient. For example, if a clinician tells an Asian American patient outright that he or she is diagnosed with major depressive disorder, Dr. Yeung estimated that approximately one-half of patients will never return for treatment. For such reasons, this practice of selectively withholding information is acceptable and even standard procedure in Asian mental health systems. Since such a practice is prohibited by Western medical regulations, a difficult question of ethics arises: Is one obligated to disclose all diagnostic information to a patient, even if one suspects that it will negatively impact the patients mental health (which is likely already somewhat unstable), and potentially prevent him or her from continuing treatment?

There is a definite need for further research in this area, as many questions remain unclear. What psychiatric and psychological terminology should be used with Asian Americans, and what diagnostic terms are most stigmatized and wrongly interpreted by the patient? How should mental health professionals help Asian Americans to better conceptualize mental illness, dispelling negative stereotypes? Do the treatment approaches need to be modified from the traditional Western framework, and what techniques are the most acceptable and effective with Asian 
Americans? Especially in Boston (as most studies in this field are conducted Los Angeles), there is a lack of research on issues such as retention rates, service outcomes, satisfaction with care, and community attitudes toward mental illness and mental health services.

\section{MENTAL HEALTH AWARENESS AT BOSTON COLLEGE}

Many studies have found that knowledge of and attitudes toward mental illness are strong predictors of help-seeking behaviors. ${ }^{x x x v}$ Asian American students often are found to have more negative attitudes toward mental illness in addition to decreased rates of mental health service utilization. xxxvi Given the high incidence of mental illness in today's society, and the reported hesitancy of students to

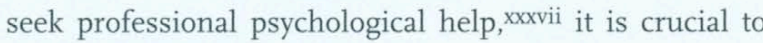
continually monitor public beliefs regarding mental illness if there are to be gains in prevention, early intervention, self-help techniques, and community support of those with mental illness.

To obtain more information about mental health issues on the Boston College campus, I interviewed Dr. Frieda Wong, a clinician at BC's University Counseling Services (UCS). Dr. Wong, although only able to speak from personal experience, indicated that she had not noticed disproportionate underutilization of UCS among Asian Americans or other students of color. It was her opinion that barriers to mental health service utilization exist for all students, and did not suspect that such barriers were greatly pronounced for minority students at BC. Although no formal research has been conducted on the topic, she suggested that potential similarities in attitudes, knowledge, and help-seeking behaviors between Asian American and White American students might be a result of relatively high levels of acculturation among Asian American BC students.

\section{MENTAL HEALTH ATTITUDES SURVEY}

In an attempt to further investigate this issue, I designed a brief Mental Health Attitudes Survey to get a small sample of the knowledge and opinions of mental illness and pro- clivity toward seeking psychological help among BC students. The sample was composed of nine Asian American students and nine White American students, all sophomores and juniors of mixed gender. When presented with a classical depression vignette, both groups of students indicated that it was "somewhat" or "very likely" that the individual was experiencing a mental illness, and all students correctly identified the individual as depressed. It was noted that for many survey items there were few or no observable differences between the two racial groups. However it was found that slightly more Asian American students believed there are certain problems which should not be discussed outside of one's immediate family, and that mental illness carries with it a burden of shame.

There was a notable difference between the two ethnic groups regarding desired social distance. Participants were asked to indicate, using a 5-point scale ( $\mathrm{o}=\mathrm{Yes}, 4=\mathrm{No}$ ), the likelihood that they would engage in specific interactions with the depressed individual designed to measure social distance. As illustrated in Figure I, Asian Americans scored significantly higher than whites on every item (indicating that they desired greater social distance). In interpreting these results it is important to keep in mind that these questions were referencing an individual with a classical, nonchronic, non-psychotic presentation of depression, who as stated has only been feeling depressed for the past two weeks. Specifically of interest was the observation that the majority of Asian Americans reported they would not be willing to have the individual to marry into the family, while the majority of White Americans responded with either "Probably Yes" or "Maybe." Additionally, more than half of the White Americans would be willing to spend an evening socializing with the individual, and would be willing to make friends with the individual. Whereas in sharp contrast, none of the Asian Americans responded with "Yes" to these items, and only a few responded with "Probably Yes."

When asked about University Counseling Services, it was found that White American students were more familiar with the types of services UCS provides and felt more com- 
fortable with the idea of seeking help at UCS. It was also observed that although one-third of students in both racial groups agreed they would feel uneasy going to a psychiatrist, less than half of White American students and only one Asian American student reported feeing comfortable seeking help from UCS. Reasons cited for this hesitancy ranged from general feelings of discomfort, fears about confidentiality issues and lack of trust, lack of knowledge, and a desire to deal with personal problems on one's own. The results of this preliminary survey suggested that among BC students, although there are many similarities between Asian American and White American students, the two groups differ in a few key areas. The findings of higher levels of desired social distance, greater association of mental illness with shame, and more pronounced hesitancy to utilize UCS all confirm the literature findings of lower help-seeking behaviors and negative and stigmatized attitudes towards mental illness. The results also indicated that there may be a lack of knowledge and negative misconceptions among students regarding UCS, both of which likely contribute to an observed hesitancy to utilize its services. It is important to keep in mind that this study had a very small sample size and these results are only tentative. However this data has provided a good jumping off point for future research in the upcoming year regarding knowledge and opinions of mental health issues among Asian and White American BC students.

\section{ENDNOTES}

i U.S. Census Bureau (2000)

ii Report of the Surgeon General (200I)

iii Lin \& Cheung (I999)

iv Schoen et al. (1998)

v Centers for Disease Control and Prevention (200I)

vi Liu (I990)

vii Herrick \& Brown (1998)

viii Herrick \& Brown (I998)

ix Durvasula \& Sue (1996)

$\mathrm{x}$ Lin \& Cheung (I999)

xi Kung (2003)

xii Report of the Surgeon General (200I)

xiii Leong \& Lau (200I)

xiv Kim et al. (I999)

xv Leong \& Lau (200I)

xvi Sue

xvii Leong \& Lau (200I)

xviii Leong \& Lau (200I); Chun et al. (1996)

xix Lin \& Cheung (1999)

xx Masuda et al. (I980)

xxi Report of the Surgeon General (200I)

xxii Lin \& Cheung (1999)

xxiii Zheng

xxiv Lin \& Cheung (1999)

xxv Flaherty et al. (1988)

xxvi Lopez (1989)

xxvii Root (I998)

xxviii Report of the Surgeon General (2001)

xxix Ying \& Miller (1992)

$\mathrm{xxx}$ Atkinson \& Gim (1989)

xxxi Takeuchi et al. (I995); Flaskerud \& Hu (I994); Yeh,

Takeuchi, \& Sue (1994)

xxxii Yeung \& Kung (2004)

xxxiii Lin \& Smith (2000)

xxxiv Root (I998)

xxxv Jorm (2000)

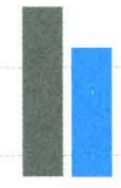

MOVE NEXT DOOR TO

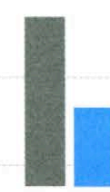

SPEND EVENINC SOCIALIZING WITH

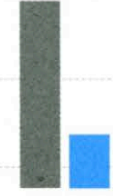

MAKE FRIENDS WITH

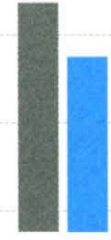

WORK CLOSELY WITH

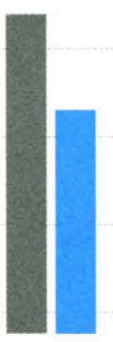

MARRY INTO FAMILY
Desired social distance for depression vignette 
xxxvi Narikiyo \& Kameoka (1992); Suan \& Tyler (1990);

Sue (1994)

xxxvii Kessler et al. (1994)

\section{REFERENCES}

Atkinson, D., Gim, R. I989. "Asian-American cultural identity and attitudes toward mental health services." Journal of Counseling Psychology, 36: pp. 209-2I2.

Centers for Disease Control and Prevention, National Center for Health Statistics. 200I. Health, United States. Hyattsville, MD: U.S. Public Health Service.

Cheung, F. \& Lau, B. rg82. "Situational variations of help-seeking behavior among Chinese patients." Comprehensive Psychiatry, 23: pp. 252-262.

Cheung, F., Lee, S., Chan, Y. 1983 . "Variations in problem conceptualizations and intended solutions among Hong Kong students." Culture, Medicine and Psychiatry, 7: pp. 263-278.

Chun, C., Enomoto, K., Sue, S. I996. "Health care issues among Asian Americans: Implications of somatization." In P.M. Kato \& T. Mann (Eds.), Handbook of diversity issues in health psychology, pp. 347-366. New York: Plenum.

Durvasula, R. \& Sue, S. 1996. "Severity of disturbance among Asian American outpatients." Cultural Diversity and Mental Health, 2: pp. 43-52.

Flaherty, J., Gaviria, F., Pathak, D., et al. 1988. “Developing instruments for cross-cultural psychiatric research." Journal of Nervous and Mental Disease, 176: pp. 257-263.

Flaskerud, J. \& Hu, L. 1994. "Participation in and outcome of treatment for major depression among low income AsianAmericans." Psychiatry Research, 53: pp. 289-300.

Herrick, C., Brown, H. 1998. "Underutilization of mental health services by Asian-Americans residing in the United States." Issues in Mental Health Nursing, 19: pp. 225-240.

Jorm, A.F., Korten, A.E., Jacomb, P.A., et al. 1997. “'Mental health literacy': a survey of the public's ability to recognize mental disorders and their beliefs about the effectiveness of treatment." Medical Journal of Australia, 166: pp. 182-186.

Kessler, R., McGonagle, K., Zhao, S., Nelson, C., et al. 1994. "Lifetime and I2-month prevalence of DSM-III-R psychiatric disorders in the United States." Archives of General Psychiatry, 5r: pp. 8-ro

Kim, B., Atkinson, D., \& Yang, P. 1999. “The Asian values scale: Development, factor analysis, validation, and reliability." Journal of Counseling Psychology, 46: pp. 342-352.
Kung, W. 2003. "Chinese Americans' help seeking for emotional distress." Social Service Review, 77: pp. IIO-I34.

Leong, F., Lau, A. 200I. "Barriers to providing effective mental health services to Asian Americans." Mental Health Services Research, 3: pp. 20I-214.

Lin, K. \& Cheung, F. I999. "Mental health issues for Asian Americans." Psychiatric Services, 50: pp. 774-780.

Lin, K. \& Smith, M. 2000. "Psychopharmacotherapy in the context of culture and ethnicity." In P. Ruiz (Ed.), Ethnicity and Psychopharmacology. Washington, DC: American Psychiatric Press: pp. I-27.

Liu, W., Yu, E., Chang, C., Fernandez, M. r99o. "The mental health of Asian American teenagers: A research challenge." In Stiffman, A. \& Davis, L. Ethnic Issues in Adolescent Mental Health: pp. 92-112.

Lopez, S. 1989. "Patient variable biases in clinical judgment: Conceptual overview and methodological considerations." Psychological Bulletin, 106: pp. 184-203.

Manderscheid, R. \& Henderson, M. (Eds.). 1998. Mental health, United States: 1998. Rockveille, MD: Center for Mental Health Services.

Masuda, M., Lin, K., Tanzuma, L. I980. Adaptational problems of Vietnamese refugees: part II. life changes and perception of life events. Archives of General Psychiatry, 37: pp. 447-450.

Mental Health: Culture, Race, and Ethnicity - a Supplement to the Mental Health: A Report of the Surgeon General. 200I. Rockville, MD: U.S. Dept of Health and Human Services.

Narikiyo, T. \& Kameoka, V. 1992. "Attributions of Mental Illness and Judgments About Help Seeking Among JapaneseAmerican and White American Students." Journal of Counseling Psychology, 39: pp. 363-369

Root, M. 1998. "Facilitating psychotherapy with Asian American clients." In Atkinson, D., Morten, G., Sue, D. (Eds.). Counseling American Minorities. Boston, MA: McGraw Hill.

Schoen, C., et al. 1998. The Health of Adolescent Boys: Commonwealth Fund Survey Findings. New York: Louis Harris and Associates, Inc

Suan, L. \& Tyler, J. I990. "Mental health values and preference for mental health resources of Japanese-American and Caucasian-American students." Professional Psychology: Research and Practice, 2I: pp. 29I-296.

Sue, D. 1994. "Asian-American mental health and help-seeking behavior: comment on Solberg et al. (1994), Tata and Leong 
(1994), and Lin (1994)." Journal of Counseling Psychology, 41: pp. 292-295

Sue, D. \& Sue, S. 1987 . Counseling in the culturally different. New York: Wiley.

Sue, S. 1976. "Conceptions of mental illness among Asian and Caucasian-American students." Psychological Reports, 38 : pp. 703-708.

Takeuchi, D., Sue, S., \& Yeh, M. I995. "Return rates and outcomes from ethnicity-specific mental health programs in Los Angeles." American Journal of Public Health, 85: pp. 638 643 .

Uba, L. I982. "Meeting the mental health needs of Asian Americans: Mainstream or segregated services." Profession Psychology: Research \& Practice, 13: pp. 215-22I.

U.S. Census Bureau. 2001. The Asian and Pacific Islander Population in the United States: March 2000 (Update) (PPL-I46) Retrieved from http://www.census.gov/ population/ www/socdemo/race/api.html.

Yeh, M., Takeuchi, D., Sue, S. 1994. "Asian American children treated in mental health system: A comparison of parallel and mainstream outpatient service centers." Journal of Clinical Child Psychology, 23: pp. 5-12.

Yeung, A. \& Kung, W.2004. "How culture impacts on the treatment of mental illnesses among Asian-Americans." Psychiatric Times, pp. 34-36.

Ying, Y. \& Miller, L. I992. "Help-seeking behavior and attitude of Chinese Americans regarding psychological problems." American Journal of Community Psychology, 20: pp. 549-556.

Zhang, A., Snowden, L., Sue, S. I998. “Differences between Asian and White Americans' help seeking and utilization patterns in the Los Angeles area." Journal of Community Psychology

Zheng, Y, Lin, K., Takeuchi, D, et al. 1997. “An epidemiological study of neurasthenia in Chinese-Americans in Los Angeles." Comprehensive Psychiatry 